\title{
Screening for early onset neonatal sepsis: NICE guidance-based practice versus projected application of the Kaiser Permanente sepsis risk calculator in the UK population
}

\author{
Nitin Goel (1) , ${ }^{1,2}$ Sudeep Shrestha, ${ }^{3}$ Rhian Smith, ${ }^{4}$ Akshay Mehta, ${ }^{1}$ Malini Ketty, ${ }^{2}$ \\ Helen Muxworthy, ${ }^{5}$ Artur Abelian, ${ }^{6}$ Vickness Kirupaalar, ${ }_{1}^{7}$ Shakir Saeed, ${ }^{8}$ Shikha Jain, ${ }^{7}$ \\ Amar Asokkumar, ${ }^{2}$ Murali Natti, ${ }^{3}$ Ian Barnard, ${ }^{4}$ Prem Kumar Pitchaikani, ${ }^{9}$ \\ Sujoy Banerjee ${ }^{1}$
}

${ }^{1}$ Neonatal Medicine, Singleton Hospital, Swansea, UK

${ }^{2}$ Neonatal Medicine, University Hospital of Wales, Cardiff, UK

${ }^{3}$ Neonatal Medicine, Royal Gwent Hospital, Newport, UK ${ }^{4}$ Neonatal Medicine, Glan Clwyd Hospital, Rhyl, UK

5Women's Health, Singleton

Hospital, Swansea, UK

${ }^{6}$ Paediatrics, Wrexham Maelor

Hospital, Wrexham, UK

${ }^{7}$ Paediatrics, Princess of Wales

Hospital, Bridgend, UK

${ }^{8}$ Paediatrics, Ysbyty Gwynedd,

Bangor, UK

${ }^{9}$ Paediatrics, Glangwili General Hospital, Carmarthen, UK

Correspondence to Sujoy Banerjee, Neonatal Medicine, Singleton Hospital, Swansea SA2 80A, UK sujoybanerjee@doctors.org.uk, sujoy.banerjee@wales.nhs.uk

Received 2 January 2019 Revised 24 June 2019 Accepted 1 July 2019 Published Online First 11 July 2019

\section{SLinked}

- http://dx.doi.org/10.1136/ fetalneonatal-2019-317840

Check for updates

(C) Author(s) (or their employer(s)) 2020. No commercial re-use. See rights and permissions. Published by BMJ.

To cite: Goel N, Shrestha S, Smith R, et al. Arch Dis Child Fetal Neonatal Ed

2020;105:F118-F122.

\section{ABSTRACT}

Objective To compare management recommendations of the National Institute for Health and Care Excellence (NICE) guidelines with the Kaiser Permanente sepsis risk calculator (SRC) for risk of early onset neonatal sepsis (EONS).

Design Multicentre prospective observational projection study.

Setting Eight maternity hospitals in Wales, UK.

Patients All live births $\geq 34$ weeks gestation over a

3-month period (February-April 2018).

Methods Demographics, maternal and infant risk factors, infant's clinical status, antibiotic usage and blood culture results from first 72 hours of birth were collected. Infants were managed using NICE recommendations and decisions compared with that projected by SRC.

Main outcome measure Proportion of infants recommended for antibiotics on either tool.

Results 0 4992 eligible infants, complete data were available for 3593 (71.9\%). Of these, 576 (16\%) were started on antibiotics as per NICE recommendations compared with $156(4.3 \%)$ projected by the SRC, a relative reduction of $74 \%$. Of the 426 infants avoiding antibiotics, SRC assigned 314 (54.6\%) to normal care only. There were seven positive blood cultures - three infants were recommended antibiotics by both, three were not identified in the asymptomatic stage by either; one was a contaminant. No EONS-related readmission was reported.

Conclusion The judicious adoption of SRC in UK clinical practice for screening and management of EONS could potentially reduce interventions and antibiotic usage in three out of four term or near-term infants and promote earlier discharge from hospital in $>50 \%$. We did not identify any EONS case missed by SRC when compared with NICE. These results have significant implications for healthcare resources.

\section{INTRODUCTION}

Early onset neonatal sepsis (EONS), defined as blood or cerebrospinal fluid (CSF) culture-proven infection within 72 hours after birth, has significant morbidity and mortality. ${ }^{1}$ In high-income countries, the overall incidence of EONS is 0.5-0.9/1000 live births and 0.3-0.6/1000 live births in term and

\section{What is already known on this topic?}

Early onset neonatal sepsis (EONS) is an infrequent but serious illness and current management strategies involve screening and treatment of a large number of healthy infants with antibiotics.

- Inappropriate overuse of antibiotics can lead to microbial resistance and perinatal use has been associated with microbiome modulation and long-term adverse health outcomes.

- The adoption of sepsis risk calculator in the USA for EONS screening has safely reduced intervention and antibiotic treatment by nearly $50 \%$ in term or near-term babies.

\section{What this study adds?}

- The adoption of SRC can potentially reduce intervention and antibiotic use in three out of four term or near-term infants undergoing EONS screening in the UK.

- Judicious adoption of SRC through a programme of enhanced observations, staff and parent education could facilitate earlier discharge from hospital in $50 \%$ of infants currently treated.

near-term infants. ${ }^{2-5}$ Group B Streptococcus (GBS) and Escherichia coli are the most common causative organisms. ${ }^{134}$ The incidence of early onset GBS disease is 0.22 and $0.3 / 1000$ live births in the USA and the UK, respectively. ${ }^{34}$ Maternal GBS screening and intrapartum prophylaxis has reduced the incidence of EONS. However, an estimated $30 \%$ of all births in the USA is associated with intrapartum antibiotics. $^{2} 3$ 5-7

Implementation of universal GBS screening and the Centers for Disease Control and Prevention (CDC) guideline in the USA led to $14 \%-24 \%$ of newborns undergoing blood tests; 5\%-12\% received antibiotics. ${ }^{5} 89$ In the UK, management of EONS is influenced by the National Institute of 
Clinical Excellence (NICE) and the Royal College of Obstetricians and Gynaecologists guidance. ${ }^{10}{ }^{11}$ Hospital audits in the UK suggest that $13 \%-20 \%$ of infants on the postnatal wards receive empirical antibiotics. ${ }^{12}$ The NICE EONS guidance is a categorical model based on maternal risk factors and infant clinical indicators. It uses threshold values and dichotomous outcomes with additional weightage for specific parameters (red flags) to guide empiric antibiotic initiation in infants at risk of EONS. ${ }^{10}$ However, it fails to consider the infant's 'wellness' following birth and how that modifies the initial risk of EONS estimated from intrapartum risk factors. In near-term infants, good clinical condition at birth is associated with reduction in EONS risk by $60 \%-70 \% .{ }^{913} 14$ In isolation, tests such as $\mathrm{C}$ reactive protein (CRP) and white cell counts have poor predictive value for EONS. ${ }^{15}$

A screening strategy that leads to high volume prophylactic antibiotic use in the early postnatal period can have significant impact on healthy infants and families, drawing substantial financial and workforce resources. ${ }^{58}$ These include the trauma of repeated phlebotomies, cannulations, harm from extravasations, drug side effects and prescription errors. Parental anxiety and separation adversely affect bonding and breast feeding. ${ }^{16}$ In addition to global concerns of increasing microbial resistance, antibiotic exposure in the perinatal period is being linked to microbiome dysbiosis and diseases in later life, for example, obesity, allergies, type 2 diabetes mellitus and inflammatory bowel disease. ${ }^{17-19}$

The Kaiser Permanente (KP) group in Northern California, applied the Bayesian approach to create a multivariate model of predicting infant-specific EONS risk-the sepsis risk calculator (SRC), derived and validated from a case-control study of blood culture-proven EONS. ${ }^{9} 1320$ The SRC derives the 'sepsis risk score' (SRS) at birth by modifying the population risk with intrapartum risk factors of the specific mother-infant pair; it then incorporates the infant's clinical status to compute the final SRS. ${ }^{21}$ Although the SRS computation algorithm is evidence based, the clinical management recommendations (based on the SRS) are a consensus opinion of the KP clinicians. For example, infants with SRS $<1$ are assigned normal care, SRS 1-3 receive enhanced observation \pm bloodculture and symptomatic or SRS $>3$ are treated with antibiotics. The implementation of SRC in KP hospitals reduced antibiotic use by $50 \%$ without increasing the incidence of missed EONS or readmissions. ${ }^{9}$ Multiple centres have since consistently reported reduction in antibiotic use by $45 \%-50 \%$ following implementation of the SRC. ${ }^{52-25}$ Recently, the American Academy of Pediatrics policy has endorsed SRC for management of EONS in infants $\geq 34$ weeks gestation. ${ }^{14}$

There is no published data on the benefits of applying SRC to the UK population. Our study compared management decisions including antibiotic use for EONS screening using NICE-based guidelines with that projected through the virtual application of the SRC, in the UK population.

\section{METHODOLOGY}

The prospective study was undertaken in Wales (UK) for 3 months (1 February 2018 to 30 April 2018). Eight of the 11 maternity hospitals in Wales (excluding midwifery-led units) participated (combined annual birth rate of $25000 ; 76 \%$ of annual birth in Wales). This included three neonatal intensive care units (NICUs), a subregional NICU and four special care units (SCU). Three SCUs declined participation as they could not commit to collecting data due to staffing shortages. Singleton Hospital, Swansea held anonymised data centrally for analysis.
The project was considered as non-research/quality improvement by the institutional joint scientific research committee.

Maternal intrapartum risk factors and infant parameters were collected for each infant $\geq 34$ weeks gestation at birth. Infants were managed according to NICE guidelines. The central assessors applied the SRC on each infant retrospectively, aligning this to the timing of clinical assessment using NICE, to determine the SRS and recommendations. The clinicians in the participating centres were blinded to this information. A background EONS incidence of 0.5/1000 live births was used for computation on the SRC; the closest estimated incidence from studies of term and near-term infants in high-income countries including the UK. ${ }^{3}{ }^{1421}$ The NICE risk factors (red and non-red flags) that prompted initiation of antibiotics were recorded, ${ }^{10}$ as were positive blood cultures drawn within 72 hours of life, neonatal unit admissions and EONS readmissions within a week of birth. True EONS was defined by positive blood or CSF culture with pathogenic organism that was treated with antibiotics for at least 5 days. All culture confirmed EONS were reviewed. Statistical analysis was performed using IBM SPSS Statistics V.25.0 software. McNemar's non-parametric test was used to assess significant differences in matched proportions.

\section{RESULTS}

During the study period, 4992 infants were born at gestation $\geq 34$ weeks. Complete data were available in 3593 infants $(71.9 \%)$. Of these, $576(16 \%)$ were commenced on antibiotics as per NICE recommendations. If SRC recommendations were implemented instead, only 156 (4.3\%) infants would have received antibiotics. Table 1 compares the population characteristics, maternal risk factors and the infant's clinical status in the entire study cohort with subsets of infants commenced on antibiotics as per NICE guidelines and that projected by the SRC.

Only $150(26 \%)$ of the 576 infants who received antibiotics would have done so as per SRC recommendations. Another 51 $(8.9 \%)$ would have undergone blood cultures and enhanced observations while antibiotics were withheld; 61 (10.6\%) were recommended enhanced observations only without blood cultures or antibiotics. The remaining 314 (54.5\%) infants were assigned to normal care.

Of the 3017 infants not started on antibiotics as per NICE, 3011 would have also avoided antibiotics as per SRC $(99.8 \%$ agreement). The remaining six infants were recommended antibiotics by SRC but remained well without treatment. Therefore, 420 infants would have avoided antibiotics as per SRC (absolute difference $11.7 \%$ (95\% CI 10.64 to $12.74, \mathrm{p}<0.0001)$ ) (table 2).

Table 3 provides a breakdown of the risk categories as per NICE guidance that prompted initiation of antibiotics and the projected application of the SRC for each of these risk groups.

There were seven positive blood cultures. One blood culture was clinically classified as a contaminant (alpha-haemolytic streptococci) and the infant remained well without treatment. Three infants were correctly identified by either tool at birth and received prophylactic antibiotics appropriately. Two infants were symptomatic at a later stage and neither NICE nor SRC identified them as high-risk at birth. The remaining infant was screened at 6 hours of age for mild temperature instability only; grew Staphylococcus aureus in blood culture and treated with antibiotics for 2 weeks. The infant remained well on postnatal ward and serial CRPs were normal. This infant was identified by clinical vigilance rather than strict use of either tool. There were no EONS readmissions in the first week of birth. 
Table 1 Population characteristics of the infants included in the study

\begin{tabular}{|c|c|c|c|c|}
\hline \multicolumn{2}{|c|}{ Population characteristics of infants included } & $\begin{array}{l}\text { All infants } \\
(n=3593) \\
N(\%)\end{array}$ & $\begin{array}{l}\text { Infants on antibiotics as per } \\
\text { NICE }(n=576) \\
n(\%)\end{array}$ & $\begin{array}{l}\text { Infants on antibiotics as } \\
\text { projected by SRC ( } n=156) \\
n(\%)\end{array}$ \\
\hline \multicolumn{2}{|l|}{ Gestational age $<37$ weeks } & $252(7)$ & $136(24)$ & 59 (38) \\
\hline \multicolumn{2}{|c|}{ Rupture Of membranes $\geq 18$ hours } & $573(16)$ & $204(35)$ & $38(24)$ \\
\hline \multicolumn{2}{|c|}{ Maternal temperature $\geq 38^{\circ} \mathrm{C}$} & $194(5)$ & $161(28)$ & $26(17)$ \\
\hline \multirow[t]{3}{*}{ GBS status } & Positive & $200(5.5)$ & $68(12)$ & $15(10)$ \\
\hline & Negative & $1249(35)$ & $181(31)$ & $59(38)$ \\
\hline & Unknown & $2144(60)$ & $327(57)$ & $82(53)$ \\
\hline \multicolumn{5}{|l|}{ Maternal antibiotics } \\
\hline \multicolumn{2}{|c|}{ GBS-specific intrapartum Prophylaxis $>2$ hours prebirth } & $63(2)$ & $19(3)$ & $2(1)$ \\
\hline \multicolumn{2}{|c|}{ Broad-spectrum antibiotics 2 to $<4$ hours prebirth } & $40(1)$ & $29(5)$ & $5(3)$ \\
\hline \multicolumn{2}{|c|}{ Broad-spectrum antibiotics $>4$ hours prebirth } & $147(4)$ & $114(20)$ & $12(10)$ \\
\hline \multicolumn{2}{|c|}{ Maternal antibiotics $<2$ hours prebirth or within 24 hours postbirth } & $106(3)$ & $102(18)$ & $11(7)$ \\
\hline \multirow[t]{3}{*}{ Clinical status as per SRC } & Well & $3308(92)$ & $320(56)$ & $4(2.5)$ \\
\hline & Equivocal & $158(4.4)$ & $129(22)$ & $20(13)$ \\
\hline & Unwell & $132(3.6)$ & $127^{*}(22)$ & $132(82.5)$ \\
\hline
\end{tabular}

${ }^{*}$ Five infants were classed unwell as per SRC criteria centrally, but clinicians had chosen to observe them.

GBS, Group B Streptococcus; SRC, sepsis risk calculator.

Figure 1 is a box plot showing the distribution of the final SRS in three cohorts-all infants in the study population, infants initiated on antibiotics as per NICE and those recommended antibiotics by SRC. In the study cohort, 3297 (91.8\%) infants had final SRS score $\leq 0.5 / 1000$ live births; 100 (2.8\%) between 0.51 and $1.0,103(2.8 \%)$ between 1.01 and 3 and 93 were $>3$ (2.6\%). The median final SRS score was 0.04 (Q1 0.02-Q3 0.1) for the study cohort, 0.4 (Q1 0.11-Q3 1.46) for the infants treated with antibiotics as per NICE and 4.28 (Q1 1.61-Q3 8.13 ) for those projected for antibiotic treatment by the SRC.

\section{DISCUSSION}

Despite validation in different clinical settings, the UK clinicians are understandably cautious about incorporating the SRC in their clinical practice in the absence of any UK-based study into its effectiveness and safety. There are differences in the UK and the US approach to intrapartum screening and treatment for GBS. The UK paediatricians prefer to treat an infant with antibiotics if they trigger a threshold for blood culture, in contrast to the practice in some US centres of blood culture and close observation without antibiotic therapy. Despite these differences, there is growing uneasiness among many UK clinicians on the number of well babies exposed to unnecessary and potentially harmful antibiotics at a critical period of their life. Our study is the first multicentre prospective evaluation of the SRC in the UK population.

Table 2 Comparison of antibiotic use as per NICE guidance vs recommendations of the SRC in the study population

\begin{tabular}{|c|c|c|c|c|}
\hline & \multicolumn{2}{|c|}{ Kaiser Permanente SRC } & \multirow{2}{*}{$\begin{array}{l}\text { Total } \\
\text { (\% of study } \\
\text { cohort) }\end{array}$} \\
\hline & & No antibiotics & Antibiotics & \\
\hline \multirow{2}{*}{$\begin{array}{l}\text { Current guidance } \\
\text { (NICE) }\end{array}$} & No antibiotics & 3011 & 6 & $3017(84)$ \\
\hline & Antibiotics & 426 & 150 & $576(16)$ \\
\hline \multicolumn{2}{|c|}{ Total (\% of study cohort) } & $3437(95.7)$ & $156(4.3)$ & $3593(100)$ \\
\hline
\end{tabular}

Sixteen per cent antibiotics as per NICE compared with $4.3 \%$ as per SRC. Absolute difference $11.7 \%$ ( $95 \% \mathrm{Cl} 10.64$ to 12.74$), \mathrm{p}<0.0001$, McNemar's test for matched proportions.

NICE, National Institute for Health and Care Excellence; SRC, sepsis risk calculator.
Our study shows that adopting SRC could potentially avoid empirical antibiotics for EONS screening in three out of four infants currently treated $(11.7 \%$ of all term or near-term births, a $74 \%$ relative reduction). This is consistent with previous reports but shows a greater effect than published elsewhere. ${ }^{5} 9$ 22-25 We acknowledge that some deviation from guidance (treating or withholding) is inevitable in real-life practice, reflecting the pragmatic choice of the clinicians in treatment decisions, and is not accounted in the desktop application of the SRC. One could argue that strict adherence to NICE guidance may have avoided antibiotics in those without any defined risk factors (3.1\%) and in a proportion of infants with only one non-red flag infant clinical indicator (8.9\%); SRC recommended antibiotics in $27 \%$ of the latter group (table 3 ). Therefore, even accounting for possible deviations, $>60 \%$ of infants currently treated would have avoided antibiotics with the SRC approach. Current practices are possibly overtreating a large proportion of low-risk infants with empiric antibiotics as illustrated in the distribution of SRS in figure 1.

Table 1 offers an insight into the differences of the two approaches of managing EONS risk. Ten per cent of women in the study cohort received antibiotics in labour or within 24 hours of giving birth but accounted for $46 \%$ of infants receiving antibiotics as per NICE. In contrast, maternal antibiotics appeared somewhat protective to the infant in the SRC model; only $21 \%$ of infants projected to be on antibiotics had maternal antibiotics as a risk factor. Nearly $56 \%$ of infants treated with antibiotics as per NICE were asymptomatic or categorised as 'well appearing', compared with $2.5 \%$ on the SRC. Therefore, the estimated risk reduction by SRC in infants who remained clinically well at birth and whose mothers received antibiotics were the two major contributors to the projected decrease in antibiotic use.

Our prospective study was not designed to assess safety. For an infrequent clinical condition such as EONS, it would require a much larger retrospective cohort to determine the absolute safety of the intervention. However, retrospective studies on this issue will be limited by incomplete or missed observation data, uncertainty about the precise onset of sepsis symptoms including missed opportunities for earlier detection. Application of contemporary research, practices and standards in EONS 
Table 3 Risk categories in infants initiated on antibiotics as per NICE guidance and projected recommendations by the SRC*

\begin{tabular}{|c|c|c|c|c|c|c|}
\hline \multirow[t]{2}{*}{$\begin{array}{l}\text { Risk categories as per NICE guidance in babies } \\
\text { receiving antibiotics }\end{array}$} & \multirow[t]{2}{*}{$\begin{array}{l}N \\
(576)\end{array}$} & \multirow[t]{2}{*}{$\%$} & \multicolumn{2}{|c|}{ SRC recommendations } & \multirow[b]{2}{*}{ Culture+vitals } & \multirow[b]{2}{*}{ Antibiotics } \\
\hline & & & Normal care & Vitals & & \\
\hline Maternal risk factors - red flags only & 227 & 39.4 & 150 & 41 & 13 & 23 \\
\hline Maternal risk factors - two or more non-red flags only & 74 & 12.8 & 39 & 14 & 6 & 15 \\
\hline $\begin{array}{l}\text { Maternal risk factors-one non-red flag+infant } \\
\text { symptoms only }\end{array}$ & 88 & 15.3 & 29 & 4 & 17 & 38 \\
\hline Infant clinical indicators - red flags only & 76 & 13.2 & 22 & 0 & 8 & 46 \\
\hline $\begin{array}{l}\text { Two or more clinical non-red flags in infant only (no maternal } \\
\text { risk factors or clinical red flags) }\end{array}$ & 42 & 7.3 & 24 & 0 & 4 & 14 \\
\hline $\begin{array}{l}\text { One clinical non-red flag in infant only } \\
\text { (no maternal risk factors or clinical red flags) }\end{array}$ & 51 & 8.9 & 34 & 0 & 3 & 14 \\
\hline No clinical or maternal red or non-red flags & 18 & 3.1 & 16 & 2 & 0 & 0 \\
\hline Total & 576 & & 314 & 61 & 51 & 150 \\
\hline
\end{tabular}

${ }^{*}$ NICE recommends antibiotics if there are any red flags (maternal risk factors or infant clinical indicators) or a combination of two non-red flags either maternal or clinical or both. For a single non-red flag, NICE recommends observation at the discretion of the clinician.

NICE, National Institute for Health and Care Excellence; SRC, sepsis risk calculator.

management to a retrospective cohort is also perhaps inappropriate. Nevertheless, consistent with previously reported studies, our study showed no difference in the incidence of missed EONS in the asymptomatic stage using either screening tool. ${ }^{9}$ More importantly, both tools will miss a significant proportion of EONS in the asymptomatic state and neither can function without high-quality clinical vigilance, staff training and parent education.

Assuming an incidence of $0.5 / 1000$ live births, $15 \%$ presumptive treatment rate and $100 \%$ case detection, the numbers needed to treat one EONS in the asymptomatic phase in a mature cohort of infants ( $\geq 34$ weeks) is 1 in 300. Considering 40\%-60\% of EONS are not picked up in the asymptomatic state by any riskbased screening tool and nearly 50\% are symptomatic at birth, the actual number needed to treat will be $>1$ in $600 .^{3926}$ There are inherent risks in creating a system that eliminates all conceivable risks for 'presumed safety' and clinicians must decide if treating the odd case of neonatal sepsis in the early symptomatic phase outweighs the risk of subjecting 600 well babies to unnecessary interventions with potential implication on their long-term health. An effective screening tool should empirically treat fewer babies and detect all true EONS cases.

There are good scientific arguments indicating that the SRC experience in the USA should be replicable in the UK. The casecontrol data used in modelling SRC incorporate clinical practice both before and after the introduction of the CDC guidance on GBS screening. The incidence of EONS and early onset GBS disease is now similar in both countries. ${ }^{2-4}$ Irrespective of the

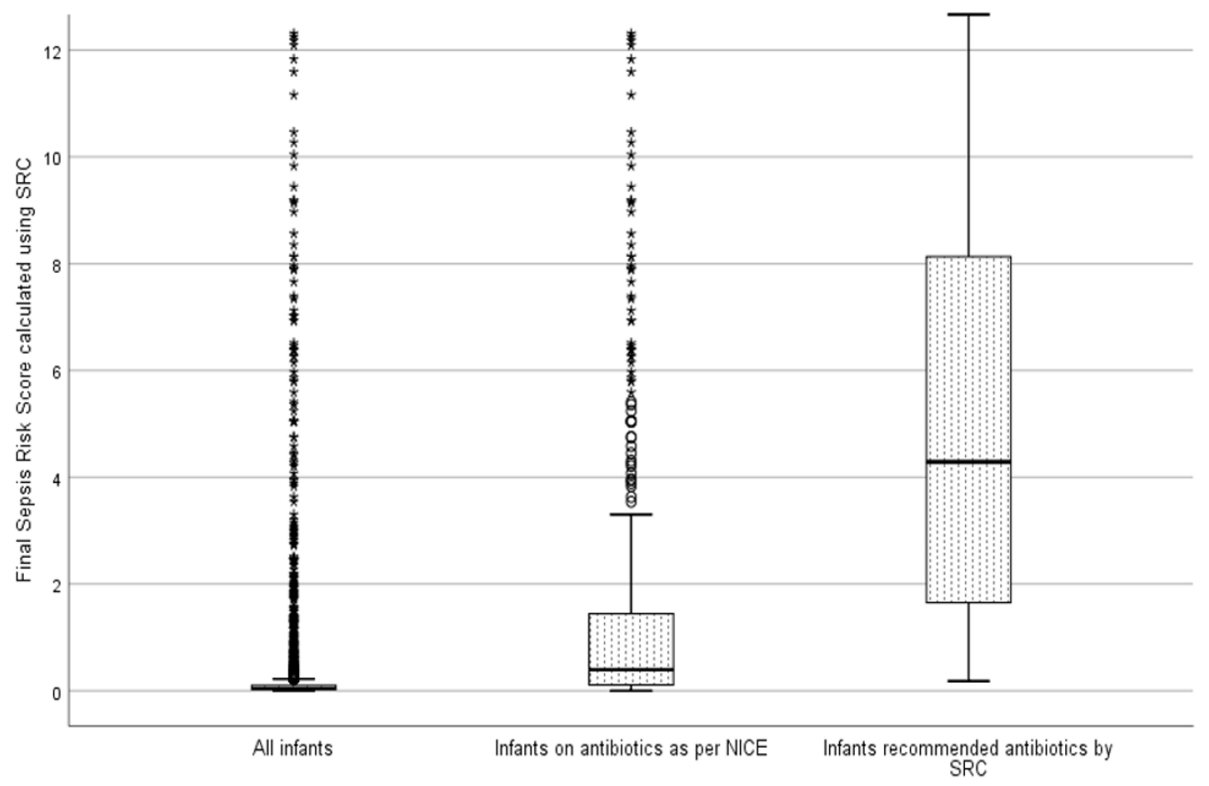

Figure 1 Box and Whisker plot showing the distribution of the final sepsis risk score (SRS) calculated using the sepsis risk calculator (SRC) in three groups-all infants in the study (median SRS of 0.04), infants on antibiotics as per the National Institute for Health and Care Excellence (NICE) (median SRS of 0.4) and infants recommended antibiotics by the SRC (median SRS of 4.28). Each box depicts the IQR, the line within the box represents the median while the lower and upper margins of the box represent the 25 th and the 75 th centile, respectively. The whiskers extend 1.5 times the IQR from the median. The circles (o) represent outliers (1.5 to $\leq 3$ times the IQR from median) and the asterisk $\left({ }^{*}\right)$ plot the extreme outliers ( $>3$ times the IQR from median). The SRS score in the study population was low; infants treated with antibiotics as per NICE had lower SRS compared with infants recommended antibiotics by the SRC. 
screening strategy (risk-based or laboratory-based), intrapartum GBS prophylaxis is highly effective. Appropriate intrapartum management is more important than GBS status itself. In fact, GBS status (positive, negative or unknown) contributes to only $2.3 \%$ of the multivariate model underpinning the SRC. ${ }^{20}$

The SRC assigned 'normal care' to $54.7 \%$ infants who received antibiotics as per NICE. We should apply caution and prudence before recommending early discharge for all these babies. The duration of routine hospital stay, postnatal care and staffing profile in the USA is different to that in the National Health Service. Staffing on the postnatal ward in the UK is frequently suboptimal and oriented to maternal care; many smaller hospitals also lack transitional care facilities. We would advise close observation of these babies for 12-24 hours in hospital during which most EONS symptoms are likely to manifest. High-quality written information and education for staff and parents will be particularly important to maintain professional and public confidence in the early implementation phase. The resources required to monitor babies would be far less than required to treat with antibiotics, will lead to earlier discharge and improve patient flow through labour wards, postnatal wards and neonatal units. If SRC recommends enhanced observation, babies should remain in hospital for close observation without treatment for 24-36 hours. Where blood culture is advised, UK clinicians may prefer to treat this small number of relatively high-risk infants while awaiting results.

Our study had complete data for $71.9 \%$ of eligible infants. The study relied on voluntary reporting by routine clinical staff and highlights the challenges of undertaking a multicentre prospective study without formal funding and amidst significant staffing shortages. However, we are confident that no EONS case was missed as we had complete data on positive blood cultures, neonatal admissions and readmissions. The proportions of sick infants admitted to neonatal units in the study cohort were not largely different to that for the missing data, suggesting equal case mix. We have not addressed the issue of culture negative sepsis in this paper, which traditionally relies either on clinical suspicion or retrospective designation by non-specific tools such as CRP. Currently, culture-negative sepsis is overdiagnosed, and a period of close observation and clinical scrutiny should identify most cases.

\section{CONCLUSIONS}

Compared with the current UK practice, the judicious adoption of SRC could potentially reduce interventions and antibiotic usage in nearly three out of four term and near-term infants and promote earlier discharge in at least half of these infants, without missing additional cases of EONS. It can be a good example of prudent and translatable healthcare practice with potential to avoid unnecessary interventions in a large proportion of low-risk newborns and release precious healthcare resources.

Acknowledgements The authors would like to thank many junior doctors, advanced nurse practitioners and midwives who helped with routine data collection in a busy clinical environment. The authors would like to thank Dr Emily Clark, Dr Damitha Ratnasinghe and Dr Brendan Harrington for contributing in different stages of the study. The authors would like to thank Professor Karen Puopolo of Children's Hospital of Philadelphia, USA for her advice and practical guidance on using the sepsis risk calculator. The authors would also like to thank the Kaiser Permanente Group for allowing free access to the neonatal sepsis risk calculator.

Contributors SB conceived the study. NG and SB designed the study protocol, data collection tools, analysed the data, drafted the manuscript and revised this for publication. SS, RS, AM, MK, HM, AA, VK, SS, SJ, AA, MN, IB and PP coordinated and collated data, critically appraised and approved the manuscript.
Funding This research received no specific grant from any funding agency in the public, commercial or not-for-profit sectors.

Competing interests None declared.

Patient consent for publication Not required.

Provenance and peer review Not commissioned; externally peer reviewed.

ORCID iD

Nitin Goel http://orcid.org/0000-0002-9718-0882

\section{REFERENCES}

1 Weston EJ, Pondo T, Lewis MM, et al. The burden of invasive early-onset neonatal sepsis in the United States, 2005-2008. Pediatr Infect Dis J 2011;30:937-41.

2 Schrag SJ, Verani JR. Intrapartum antibiotic prophylaxis for the prevention of perinatal group B streptococcal disease: experience in the United States and implications for a potential group B streptococcal vaccine. Vaccine 2013;31(Suppl 4):D20-6.

3 Cailes B, Kortsalioudaki C, Buttery J, et al. Epidemiology of UK neonatal infections: the neonIN infection surveillance network. Arch Dis Child Fetal Neonatal Ed 2018;103:F5 47-F553.

4 Schrag SJ, Farley MM, Petit S, et al. Epidemiology of Invasive Early-Onset Neonatal Sepsis, 2005 to 2014. Pediatrics 2016;138(6):e20162013.

5 Dhudasia MB, Mukhopadhyay S, Puopolo KM. Implementation of the Sepsis Risk Calculator at an Academic Birth Hospital. Hosp Pediatr 2018;8:243-50.

6 Verani JR, McGee L, Schrag SJ. Division of Bacterial Diseases, National Center for Immunization and Respiratory Diseases, Centers for Disease Control and Prevention (CDC). Prevention of perinatal group B streptococcal disease-revised guidelines from CDC, 2010. MMWR Recomm Rep 2010;59(RR-10):1-36.

7 Van Dyke MK, Phares CR, Lynfield R, et al. Evaluation of universal antenatal screening for group B streptococcus. N Engl J Med 2009;360:2626-36.

8 Mukhopadhyay S, Dukhovny D, Mao W, et al. 2010 perinatal GBS prevention guideline and resource utilization. Pediatrics 2014;133:196-203.

9 Kuzniewicz MW, Puopolo KM, Fischer A, et al. A Quantitative, Risk-Based Approach to the Management of Neonatal Early-Onset Sepsis. JAMA Pediatr 2017;171:365-71.

10 Clinical guideline [CG149]. Neonatal infection (early onset): antibiotics for prevention and treatment. $2012 \mathrm{https}: / /$ www.nice.org.uk/guidance/cg149.

11 RCOG. Group B Streptococcal Disease, Early-onset (Green-top Guideline No. 36). 2017 https://www.rcog.org.uk/en/guidelines-research-services/guidelines/gtg36/.

12 Davidson SL, King R, Greig R, et al. The Kaiser-Permanente early onset sepsis calculator - a tool to reduce antibiotic use in the UK? Neonatal Society 2016. Summer Meeting http://www.neonatalsociety.ac.uk/abstracts/davidsonsl_2016_ kaiserpermanente.shtml

13 Escobar GJ, Puopolo KM, Wi S, et al. Stratification of risk of early-onset sepsis in newborns $\geq 34$ weeks' gestation. Pediatrics 2014;133:30-6.

14 Puopolo KM, Benitz WE, Zaoutis TE. Committee On Fetus And Newborn, Committee On Infectious Diseases. Management of Neonates Born at $\geq 35$ 0/7 Weeks' Gestation With Suspected or Proven Early-Onset Bacterial Sepsis. Paediatrics. Volume 142, number 2018:6:e20182894.

15 Mukhopadhyay S, Puopolo KM. Risk assessment in neonatal early onset sepsis. Semin Perinatol 2012:36:408-15.

16 Mukhopadhyay S, Lieberman ES, Puopolo KM, et al. Effect of early-onset sepsis evaluations on in-hospital breastfeeding practices among asymptomatic term neonates. Hosp Pediatr 2015;5:203-10.

17 Murk W, Risnes KR, Bracken MB. Prenatal or early-life exposure to antibiotics and risk of childhood asthma: a systematic review. Pediatrics 2011;127:1125-38.

18 Saari A, Virta LJ, Sankilampi U, et al. Antibiotic exposure in infancy and risk of being overweight in the first 24 months of life. Pediatrics 2015;135:617-26.

19 Corvaglia L, Tonti G, Martini S, et al. Influence of intrapartum antibiotic prophylaxis for group B streptococcus on gut microbiota in the first month of life. J Pediatr Gastroenterol Nutr 2016;62:304-8

20 Puopolo KM, Draper D, Wi S, et al. Estimating the probability of neonatal early-onset infection on the basis of maternal risk factors. Pediatrics 2011;128:e1155-e1163.

21 Kaiser Permanent Research. Probability of Neonatal Early-Onset Sepsis Based on Maternal Risk Factors and the Infant's Clinical Presentation. https://neonatalsepsisca Iculator.kaiserpermanente.org/.

22 Warren S, Garcia M, Hankins C. Impact of neonatal early-onset sepsis calculator on antibiotic use within two tertiary healthcare centers. J Perinatol 2017:37:394-7.

23 Kerste M, Corver J, Sonnevelt MC, et al. Application of sepsis calculator in newborns with suspected infection. J Matern Fetal Neonatal Med 2016;29:3860-5.

24 Strunk T, Buchiboyina A, Sharp M, et al. Implementation of the Neonatal Sepsis Calculator in an Australian Tertiary Perinatal Centre. Neonatology 2018;113:379-82.

25 Achten NB, Dorigo-Zetsma JW, van der Linden PD, et al. Sepsis calculator implementation reduces empiric antibiotics for suspected early-onset sepsis. Eur J Pediatr 2018;177:741-6.

26 Polin RA. Committee on Fetus and Newborn. Management of neonates with suspected or proven early-onset bacterial sepsis. Pediatrics 2012;129:1006-15. 